\title{
Prompt Photon Production in Photoproduction at HERA
}

\author{
Krzysztof Nowak and Katharina Müller \\ Univeristy of Zürich \\ Winterthurerstrasse 190, CH - 8057 Zürich - Switzerland
}

\begin{abstract}
A measurement of prompt photons in photoproduction in ep collisions with the H1 detector is presented. The analysis is based on data taken in the years 2004-2007, corresponding to a total integrated luminosity of $340 \mathrm{pb}^{-1}$. The photon signal is extracted using a multivariate analysis based on cluster calorimeter shower shape variables. Inclusive and exclusive cross sections are presented as a function of the transverse energy $E_{T}^{\gamma}$ in the range 5 to $15 \mathrm{GeV}$ and the pseudorapidity $\eta^{\gamma}$ range -1.0 to 2.4 . The momentum fractions $x_{\gamma}$ and $x_{\text {proton }}$ of the incident photon and proton respectively carried by the constituents participating in the hard scattering process are measured as well. The results are compared to predictions from theoretical calculations.
\end{abstract}

\section{Introduction}

Isolated photons with high transverse momentum in the final state are a direct probe of the dynamics of the hard subprocess in ep collisions. The measurement provides complementary information to the study of jet production with different and generally lower corrections for hadronisation. The process gives access to parton density functions of both photon and proton. The ZEUS collaboration has reported measurements of prompt photon production $[2,3,4]$. The H1 collaboration has performed a measurement of prompt photon cross section both in photoproduction [5] and in deep inelastic scattering (DIS) [6]. Comparison to LO and NLO calculations showed that cross sections in DIS are significantly underestimated. In photoproduction, the inclusive cross section is slightly underestimated by the calculations but the photon plus jet cross section is described well.

The present preliminary analysis extends the phase space of the previous measurement in photoproduction towards larger pseudorapidities of the photon and to lower event inelasticities. The data used for the measurement have been collected by the H1 detector during the years 2004-2007 corresponding to a total integrated luminosity of $340 \mathrm{pb}^{-1}$, an increased of a factor of three over previous measurement.

\section{Experimental Method}

Photons are reconstructed as a compact electromagnetic cluster in the liquid argon calorimeter (LAr)[7]. The main experimental difficulty is the separation of photons from neutral mesons, mainly $\pi^{0}$ or $\eta$, decaying into multi-photon final states.

Events are triggered by a high energy LAr cluster. Low $Q^{2}$ DIS events are removed by excluding events with an electron in the backward calorimeter. The inelasticity is restricted to $0.1<y_{h}=\Sigma\left(E-p_{z}\right) / 2 E_{e}<0.7$ where $E_{e}$ is the energy of the incoming electron and the sum runs over the energy $(E)$ and the longitudinal momentum $\left(p_{z}\right)$ of all final state particles. The z-position of the event vertex is required to be within $40 \mathrm{~cm}$ of the nominal vertex position. In order to correctly determine the vertex position at least two well reconstructed central tracks are required. 
Photon candidates are defined by electromagnetic clusters with transverse energy $5<$ $E_{T}^{\gamma}<15 \mathrm{GeV}$ and pseudorapidity $-1.0<\eta^{\gamma}<2.4$. Candidates close to the cracks of the calorimeter are rejected. Charged particles are removed by a combined condition based on the information from the Central Tracking Detector and the Central Inner Proportional chamber [8]. The cluster has to be compact with a transverse radius of less than $6 \mathrm{~cm}$. The photon needs to be isolated, by requiring the fraction of the transverse energy of the jet containing the photon carried by the photon, $z=E_{T}^{\gamma} / E_{T}^{\text {photon-jet }}>0.9$. The invariant mass of the cluster, when combined with the closest neighbouring electromagnetic cluster with an energy larger than $80 \mathrm{MeV}$ must be larger than $0.3 \mathrm{GeV}$. This last requirement rejects candidates from $\pi^{0}$ decays with two photons reconstructed in separated clusters.

For the exclusive selection of a photon accompanied by a jet, the selection on the transverse momentum of the jet larger than $4.5 \mathrm{GeV}$ and pseudorapidity $-1.3<\eta^{j e t}<2.4$ is performed.

For both inclusive and exclusive selections the photon signal is extracted by a multivariate analysis (MVA) [9] using the shower shape variables as input. It is based on the fact that electromagnetic showers in the calorimeter initiated by isolated photons tend to be more narrow, symmetric and tend to start off slightly deeper in the calorimeter than background showers. Six different shower shape variables are combined in a MVA producing discriminator distribution in bins of transverse energy $E_{T}^{\gamma}$ and pseudorapidity $\eta^{\gamma}$. The signal content is obtained by fitting the discriminator distributions of signal and background single particles Monte Carlo samples to the data.

\section{Results}

Differential cross sections for the production of prompt photons in photoproduction are measured for $Q^{2}<1 \mathrm{GeV}^{2}, 0.1<y_{h}<0.7$ for photons with $5<E_{T}^{\gamma}<15 \mathrm{GeV}$, pseudorapidity $-1.0<\eta^{\gamma}<2.4$ and the transverse energy fraction $z=E_{T}^{\gamma} / E_{T}^{\text {photon-jet }}>0.9$.

The selection efficiency is determined with the help of prompt photon Monte Carlo events generated with PYTHIA 6.2 [10] and simulated with a full GEANT [11] simulation of the $\mathrm{H} 1$ detector. The dominant contribution to the total error is the uncertainty of the shower shapes.

All the results are compared to two sets of calculations, both corrected to hadron level and multiple interaction effects: the collinear approach of Fontannaz-Guillet-Heinrich (FGH) $[12,13]$ and $k_{T}$-factorisation based Zotov-Lipatov (ZL) approach [14].

Figure 1 shows the single differential inclusive cross sections as a function of transverse energy and the pseudorapidity of the photon. Both calculations are slightly lower than the data most significantly at low $E_{T}^{\gamma}$.

Exclusive (photon plus jet) single differential cross sections measurement as a function of momentum fractions $x_{\gamma}^{o b s}$ and $x_{\text {proton }}^{o b s}$ (Figure 2 ) show better agreement with the calculations. The largest discrepancy arises for the highest bin in $x_{\gamma}^{o b s}$, where both calculations give different prediction, while the data do not favour any of them. 

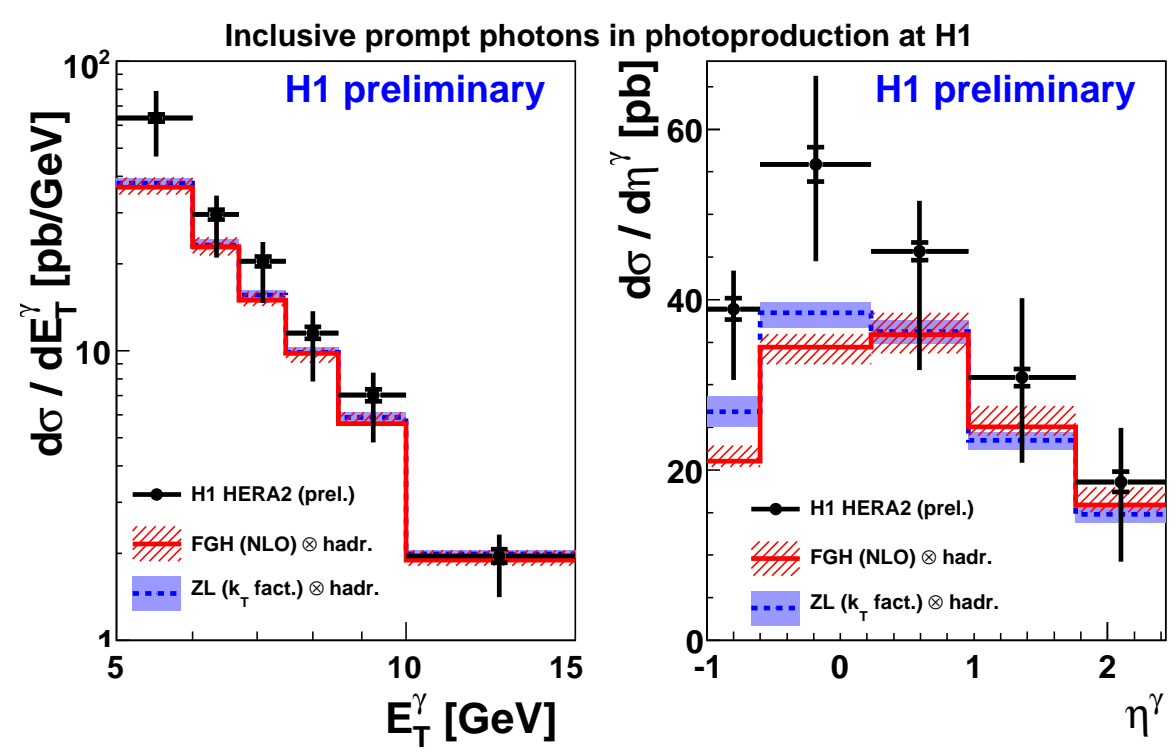

Figure 1: Inclusive prompt photon single differential cross sections as function of $E_{T}^{\gamma}$ (left) and $\eta^{\gamma}$ (right). The cross sections are measured in visible range defined by $5<E_{T}^{\gamma}<15 \mathrm{GeV}$, $Q^{2}<1 \mathrm{GeV}^{2}, 0.1<y_{h}<0.7$ and $z=E_{T}^{\gamma} / E_{T}^{\text {photon-jet }}>0.9$. The inner errors in the figures represent the statistical errors, while the outer are the statistical and the systematical errors added in quadrature. The measured cross section is compared to the Fontannaz-GuilletHeinrich (continuous red) and Zotov-Lipatov (dashed blue) calculations.

\section{Conclusions}

Both inclusive and exclusive prompt photon production in photoproduction has been measured by the H1 collaboration. The measurement has been compared to two calculations, one using a collinear approach and the other based on $k_{t}$ factorisation. The predictions for the inclusive sample are slightly lower than the data, most significantly at low $E_{T}^{\gamma}$ but there is reasonable agreement for the photon plus jet sample except at high $x_{\gamma}^{o b s}$

\section{References}

[1] Slides: http: //indico. cern. ch/contributionDisplay py? contribId=186\&sessionId=13\&conf Id=24657

[2] J. Breitweg et al. Physics Letters B 472 (2000) 1-2, 175-188 [arXiv:hep-ex/9910045]

[3] S. Chekanov et al. [ZEUS Collaboration], Phys.Lett. B511 (2001) 19-32 [arXiv:hep-ex/0104001]

[4] S. Chekanov et al. [ZEUS Collaboration], Eur.Phys.J. C49 (2007) 511-522 [arXiv:hep-ex/0608028]

[5] A. Aktas et al. [H1 Collaboration], Eur. Phys. J.C38 (2005) 437 [arXiv:hep-ex/0407018].

[6] F.D.Aaron et al. [H1 Collaboration], 0711.4578 [hep-ex].

[7] B. Andrieu et al. [H1 Calorimeter Group Collaboration], Nucl. Instrum. Meth. A336 (1993) 460.

[8] J. Becker et al., Nucl. Instrum. Meth. A 586 (2008) 190 [arXiv:physics/0701002]. 

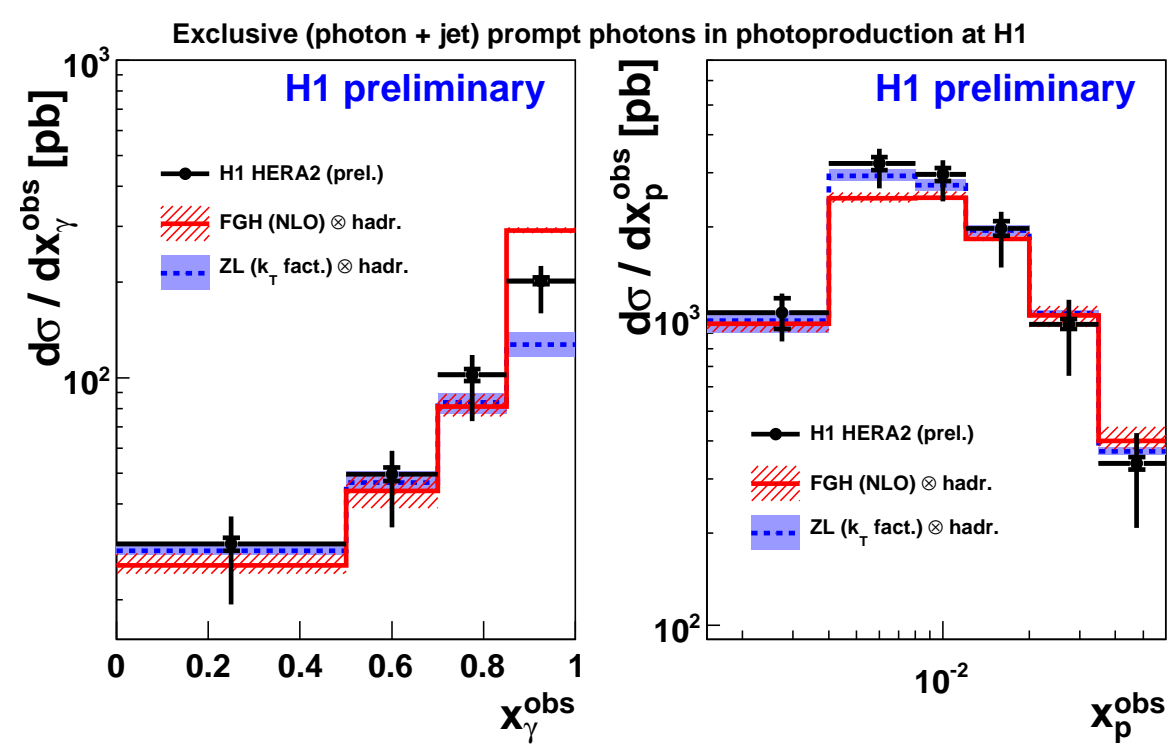

Figure 2: Exclusive prompt photon single differential cross sections as function of $x_{\gamma}^{\text {obs }}$ (left) and $x_{p}^{o b s}$ (right). The cross sections are measured in range defined in caption of Figure 1. In addition jet with transverse momentum $p_{T}^{\text {jet }}>4.5 \mathrm{GeV}$ and pseudorapidity $-1.3<\eta^{\text {jet }}<2.4$ is required. The inner errors in the figures represent the statistical errors, while the outer are the statistical and the systematical errors added in quadrature. The measured cross section is compared to the Fontannaz-Guillet-Heinrich (continuous red) and Zotov-Lipatov (dashed blue) calculations.

[9] J. Friedman, T. Hastie and R. Tibshirani, "The Elements of Statistical Learning", Springer Series in Statistics, 2001

[10] T. Sjöstrand et al., PYTHIA 6.2 Physics and Manual [hep-ph/0108264].

[11] GEANT 3, R.Brun et al., CERN_DD/EE/84-1.

[12] M. Fontannaz, J. P. Guillet and G. Heinrich, Eur. Phys. J. C 21 (2001) 303 [arXiv:hep-ph/0105121].

[13] M. Fontannaz and G. Heinrich, Eur. Phys. J. C 34, 191 (2004) [arXiv:hep-ph/0312009].

[14] A. V. Lipatov and N. P. Zotov, Phys. Rev. D 72 (2005) 054002 [arXiv:hep-ph/0506044]. 